\title{
OSCILLATION CRITERIA FOR A CLASS OF FUNCTIONAL PARABOLIC EQUATIONS
}

\author{
T. KUSANO and N. YOSHIDA
}

Received August 28, 1997 and, in revised form, December 4, 1997

\begin{abstract}
Oscillations of parabolic equations with functional arguments are studied, and sufficient conditions are derived for all solutions of certain boundary value problems to be oscillatory in a cylindrical domain. Our approach is to reduce the multi-dimensional problems to one-dimensional problems for functional differential inequalities.
\end{abstract}

\section{Introduction}

We are concerned with the oscillatory behavior of solutions of the parabolic equation with functional arguments

$$
\begin{aligned}
& \frac{\partial}{\partial t}\left(u(x, t)+\sum_{i=1}^{\ell} h_{i}(t) u\left(x, \rho_{i}(t)\right)\right) \\
& \quad-a(t) \Delta u(x, t)-\sum_{i=1}^{k} b_{i}(t) \Delta u\left(x, \tau_{i}(t)\right) \\
& \quad-c\left(x, t,\left(z_{i}[u](x, t)\right)_{i=1}^{M}\right)=f(x, t), \quad(x, t) \in \Omega \equiv G \times(0, \infty),
\end{aligned}
$$

1991 Mathematics Subject Classification. 35B05, 35R10.

Key words and phrases. Oscillation, parabolic equations, functional arguments.

ISSN 1425-6908 (c) Heldermann Verlag. 
where $G$ is a bounded domain of $\mathbf{R}^{n}$ with piecewise smooth boundary $\partial G$. It is assumed that

$$
z_{i}[u](x, t)= \begin{cases}u\left(x, \sigma_{i}(t)\right) & (i=1,2, \ldots, m), \\ \max _{s \in B_{i}(t)} u(x, s) & \left(i=m+1, m+2, \ldots, m_{1}\right), \\ \sum_{j=1}^{N_{i}} \int_{G} K_{i j}(x, t, y) \omega_{i j}\left(u\left(y, \sigma_{i j}(t)\right)\right) d y & \left(i=m_{1}+1, m_{1}+2, \ldots, M\right),\end{cases}
$$

where $B_{i}(t)\left(i=m+1, m+2, \ldots, m_{1}\right)$ are closed bounded sets of $[0, \infty)$ with the property that $\lim _{t \rightarrow \infty} \min _{s \in B_{i}(t)} s=\infty, \sigma_{i j}(t) \in C([0, \infty) ; \mathbf{R}) \quad(i=$ $\left.m_{1}+1, m_{1}+2, \ldots, M ; j=1,2, \ldots, N_{i}\right), \lim _{t \rightarrow \infty} \sigma_{i j}(t)=\infty, K_{i j}(x, t, y) \in$ $C(\bar{\Omega} \times \bar{G} ;[0, \infty))$, and $\omega_{i j}(s) \in C(\mathbf{R} ; \mathbf{R})$ are odd functions such that $\omega_{i j}(s) \geq$ 0 for $s>0$.

We note that $z_{i}[u](x, t) \geq 0(i=1,2, \ldots, M)$ in $G \times[T, \infty)$ for some $T>0$ if $u$ is eventually positive in $G \times(0, \infty)$.

We assume that :

$$
\begin{aligned}
& h_{i}(t) \in C^{1}([0, \infty) ;[0, \infty)) \quad(i=1,2, \ldots, \ell) ; \\
& a(t) \in C([0, \infty) ;[0, \infty)) ; \\
& b_{i}(t) \in C([0, \infty) ;[0, \infty)) \quad(i=1,2, \ldots, k) ; \\
& \rho_{i}(t) \in C^{1}([0, \infty) ; \mathbf{R}), \lim _{t \rightarrow \infty} \rho_{i}(t)=\infty \quad(i=1,2, \ldots, \ell) ; \\
& \tau_{i}(t) \in C([0, \infty) ; \mathbf{R}), \lim _{t \rightarrow \infty} \tau_{i}(t)=\infty \quad(i=1,2, \ldots, k) ; \\
& \sigma_{i}(t) \in C([0, \infty) ; \mathbf{R}), \lim _{t \rightarrow \infty} \sigma_{i}(t)=\infty \quad(i=1,2, \ldots, m) ; \\
& f(x, t) \in C(\bar{\Omega} ; \mathbf{R}) .
\end{aligned}
$$

Moreover, we assume that :

$$
\begin{aligned}
& c\left(x, t,\left(\xi_{i}\right)_{i=1}^{M}\right) \in C\left(\bar{\Omega} \times \mathbf{R}^{M} ; \mathbf{R}\right), \\
& c\left(x, t,\left(\xi_{i}\right)_{i=1}^{M}\right) \geq \sum_{i=1}^{m} p_{i}(t) \varphi_{i}\left(\xi_{i}\right) \quad \text { for } \quad\left(x, t,\left(\xi_{i}\right)_{i=1}^{M}\right) \in \Omega \times[0, \infty)^{M}, \\
& c\left(x, t,\left(-\xi_{i}\right)_{i=1}^{M}\right) \leq-\sum_{i=1}^{m} p_{i}(t) \varphi_{i}\left(\xi_{i}\right) \quad \text { for } \quad\left(x, t,\left(\xi_{i}\right)_{i=1}^{M}\right) \in \Omega \times[0, \infty)^{M},
\end{aligned}
$$

where $[0, \infty)^{j}=[0, \infty) \times[0, \infty)^{j-1}(j=1,2, \ldots, M), p_{i}(t) \in C([0, \infty) ;[0, \infty))$, $\varphi_{i}(s) \in C([0, \infty) ;[0, \infty))$, and $\varphi_{i}(s)$ are convex in $(0, \infty)(i=1,2, \ldots, m)$.

The boundary conditions to be considered are the following :

$\left(\mathrm{B}_{1}\right) u=\psi \quad$ on $\quad \partial G \times(0, \infty)$,

$\left(\mathrm{B}_{2}\right) \frac{\partial u}{\partial \nu}-\mu u=\tilde{\psi} \quad$ on $\quad \partial G \times(0, \infty)$,

where $\psi, \tilde{\psi} \in C(\partial G \times(0, \infty) ; \mathbf{R}), \mu \in C(\partial G \times(0, \infty) ;[0, \infty))$ and $\nu$ denotes the unit exterior normal vector to $\partial G$. 
Definition 1. By a solution of the boundary value problems (1), $\left(\mathrm{B}_{i}\right)$ $(i=1,2)$, we mean a function $u(x, t) \in C^{2}\left(\bar{G} \times\left[t_{-1}, \infty\right) ; \mathbf{R}\right) \cap C^{1}(\bar{G} \times$ $\left.\left[\tilde{t}_{-1}, \infty\right) ; \mathbf{R}\right) \cap C\left(\bar{G} \times\left[\hat{t}_{-1}, \infty\right) ; \mathbf{R}\right)$ which satisfies $(1),\left(\mathrm{B}_{i}\right)(i=1,2)$, where

$$
\begin{aligned}
& t_{-1}=\min \left\{0, \min _{1 \leq i \leq k}\left\{\inf _{t \geq 0} \tau_{i}(t)\right\}\right\}, \\
& \tilde{t}_{-1}=\min \left\{0, \min _{1 \leq i \leq \ell}\left\{\inf _{t \geq 0} \rho_{i}(t)\right\}\right\}, \\
& \hat{t}_{-1}=\min \left\{0, \min _{1 \leq i \leq m}\left\{\inf _{t \geq 0} \sigma_{i}(t)\right\}, \min _{\substack{m_{1}+1 \leq i \leq M \\
1 \leq j \leq N_{i}}}\left\{\inf _{t \geq 0} \sigma_{i j}(t)\right\}\right\} .
\end{aligned}
$$

Definition 2. A solution $u$ of the boundary value problems (1), $\left(\mathrm{B}_{i}\right)(i=$ $1,2)$ is said to be oscillatory in $\Omega$ if $u$ has a zero in $G \times(t, \infty)$ for any $t>0$.

In 1983, Bykov and Kultaev [2] have studied the oscillations of functional parabolic equations including the special case of (1). Oscillation theory for functional parabolic equations has been extensively developed in recent years by several authors; see, for example, [4-6, 8-10]. However, most of the papers except $[2,9]$ pertain to the parabolic equations (1) with $-c\left(x, t,\left(z_{i}[u](x, t)\right)_{i=1}^{M}\right)$ replaced by $c\left(x, t,\left(z_{i}[u](x, t)\right)_{i=1}^{M}\right)$. We mention in particular the paper [1] which deals with impulsive nonlinear parabolic equations.

The purpose of this paper is to derive sufficient conditions for every solution $u$ of the boundary value problems $(1),\left(\mathrm{B}_{i}\right)(i=1,2)$ to be oscillatory in $\Omega$. In Section 2 we reduce the multi-dimensional problems to onedimensional oscillation problems. Section 3 is devoted to the nonexistence of eventually positive solutions of the associated functional differential inequalities. In Section 4 we combine the results of Sections 2 and 3 to obtain various oscillation results for the functional parabolic equation (1).

\section{Reduction to functional differential inequalities}

In this section we show that the boundary value problems $(1),\left(\mathrm{B}_{i}\right)(i=$ $1,2)$ can be reduced to one-dimensional oscillation problems.

It is known that the smallest eigenvalue $\lambda_{1}$ of the eigenvalue problem

$$
\begin{aligned}
-\Delta w & =\lambda w \text { in } G, \\
w & =0 \text { on } \partial G
\end{aligned}
$$

is positive and the corresponding eigenfunction $\Phi(x)$ may be chosen so that $\Phi(x)>0$ in $G$ (see Courant and Hilbert [3]). 
We use the notation :

$$
\begin{aligned}
& F(t)=\int_{G} f(x, t) \Phi(x) d x \cdot\left(\int_{G} \Phi(x) d x\right)^{-1}, \\
& \Psi(t)=\int_{\partial G} \psi \frac{\partial \Phi}{\partial \nu}(x) d S \cdot\left(\int_{G} \Phi(x) d x\right)^{-1}, \\
& \tilde{F}(t)=\frac{1}{|G|} \int_{G} f(x, t) d x \\
& \tilde{\Psi}(t)=\frac{1}{|G|} \int_{\partial G} \tilde{\psi} d S
\end{aligned}
$$

where $|G|=\int_{G} d x$.

We define the function spaces $\mathcal{B}_{\gamma}(\Omega)$ and $\tilde{\mathcal{B}}_{\Gamma}$ by

$$
\begin{aligned}
& \mathcal{B}_{\gamma}(\Omega)=\left\{u(x, t) \in C^{2}(\bar{\Omega} ; \mathbf{R}) ;|u(x, t)| \leq \gamma(x, t) \text { on } \bar{\Omega}\right\}, \\
& \tilde{\mathcal{B}}_{\Gamma}=\left\{y(t) \in C^{1}\left(\left[T_{y}, \infty\right) ; \mathbf{R}\right) ;|y(t)| \leq \Gamma(t) \text { on }\left[T_{y}, \infty\right)\right\},
\end{aligned}
$$

where $T_{y}$ is a positive constant depending on $y(t), \gamma(x, t)$ is a positive continuous function on $\bar{\Omega}$, and

$$
\Gamma(t)=\int_{G} \gamma(x, t) \Phi(x) d x \cdot\left(\int_{G} \Phi(x) d x\right)^{-1} .
$$

Theorem 1. If the functional differential inequalities

$$
\begin{aligned}
& \frac{d}{d t}\left(y(t)+\sum_{i=1}^{\ell} h_{i}(t) y\left(\rho_{i}(t)\right)\right)+\lambda_{1} a(t) y(t) \\
& +\lambda_{1} \sum_{i=1}^{k} b_{i}(t) y\left(\tau_{i}(t)\right)-\sum_{i=1}^{m} p_{i}(t) \varphi_{i}\left(y\left(\sigma_{i}(t)\right)\right) \geq \pm G(t)
\end{aligned}
$$

have no eventually positive solutions of class $\tilde{\mathcal{B}}_{\Gamma}$, then every solution $u \in$ $\mathcal{B}_{\gamma}(\Omega)$ of the boundary value problem $(1),\left(B_{1}\right)$ is oscillatory in $\Omega$, where

$$
G(t)=F(t)-a(t) \Psi(t)-\sum_{i=1}^{k} b_{i}(t) \Psi\left(\tau_{i}(t)\right) .
$$

Proof. Suppose to the contrary that there exists a solution $u \in \mathcal{B}_{\gamma}(\Omega)$ of the problem (1), $\left(\mathrm{B}_{1}\right)$ which is nonoscillatory in $\Omega$. First we assume that $u>0$ in $G \times\left[t_{0}, \infty\right)$ for some $t_{0}>0$. The hypothesis implies that

$$
c\left(x, t,\left(z_{i}[u](x, t)\right)_{i=1}^{M}\right) \geq \sum_{i=1}^{m} p_{i}(t) \varphi_{i}\left(u\left(x, \sigma_{i}(t)\right)\right) \quad \text { in } \quad G \times\left[t_{1}, \infty\right)
$$


for some $t_{1} \geq t_{0}$. Hence, from (1) we see that

$$
\begin{aligned}
& \frac{\partial}{\partial t}\left(u(x, t)+\sum_{i=1}^{\ell} h_{i}(t) u\left(x, \rho_{i}(t)\right)\right) \\
& \quad-a(t) \Delta u(x, t)-\sum_{i=1}^{k} b_{i}(t) \Delta u\left(x, \tau_{i}(t)\right) \\
& \quad-\sum_{i=1}^{m} p_{i}(t) \varphi_{i}\left(u\left(x, \sigma_{i}(t)\right)\right) \geq f(x, t) \quad \text { in } \quad G \times\left[t_{1}, \infty\right) .
\end{aligned}
$$

Multiplying (3) by $\Phi(x) \cdot\left(\int_{G} \Phi(x) d x\right)^{-1}$ and then integrating over $G$ yields

$$
\begin{aligned}
& \frac{d}{d t}\left(U(t)+\sum_{i=1}^{\ell} h_{i}(t) U\left(\rho_{i}(t)\right)\right)-a(t) K_{\Phi} \int_{G} \Delta u(x, t) \Phi(x) d x \\
& \quad-\sum_{i=1}^{k} b_{i}(t) K_{\Phi} \int_{G} \Delta u\left(x, \tau_{i}(t)\right) \Phi(x) d x \\
& \quad-\sum_{i=1}^{m} p_{i}(t) K_{\Phi} \int_{G} \varphi_{i}\left(u\left(x, \sigma_{i}(t)\right)\right) \Phi(x) d x \\
& \geq F(t), \quad t \geq t_{1},
\end{aligned}
$$

where

$$
\begin{aligned}
& K_{\Phi}=\left(\int_{G} \Phi(x) d x\right)^{-1}, \\
& U(t)=\int_{G} u(x, t) \Phi(x) d x \cdot\left(\int_{G} \Phi(x) d x\right)^{-1} .
\end{aligned}
$$

It follows from Green's formula that

$$
\begin{aligned}
& K_{\Phi} \int_{G} \Delta u(x, t) \Phi(x) d x \\
= & K_{\Phi} \int_{\partial G}\left[\frac{\partial u}{\partial \nu}(x, t) \Phi(x)-u(x, t) \frac{\partial \Phi}{\partial \nu}(x)\right] d S+K_{\Phi} \int_{G} u(x, t) \Delta \Phi(x) d x \\
= & -K_{\Phi} \int_{\partial G} \psi \frac{\partial \Phi}{\partial \nu}(x) d S-\lambda_{1} K_{\Phi} \int_{G} u(x, t) \Phi(x) d x \\
= & -\Psi(t)-\lambda_{1} U(t), \quad t \geq t_{1} .
\end{aligned}
$$

Analogously we have

$$
K_{\Phi} \int_{G} \Delta u\left(x, \tau_{i}(t)\right) \Phi(x) d x=-\Psi\left(\tau_{i}(t)\right)-\lambda_{1} U\left(\tau_{i}(t)\right), \quad t \geq t_{2}
$$


for some $t_{2} \geq t_{1}$. Applying Jensen's inequality [7, p.160], we obtain

$$
K_{\Phi} \int_{G} \varphi_{i}\left(u\left(x, \sigma_{i}(t)\right)\right) \Phi(x) d x \geq \varphi_{i}\left(U\left(\sigma_{i}(t)\right)\right), \quad t \geq t_{2} .
$$

Combining (4)-(7) yields

$$
\begin{aligned}
& \frac{d}{d t}\left(U(t)+\sum_{i=1}^{\ell} h_{i}(t) U\left(\rho_{i}(t)\right)\right)+\lambda_{1} a(t) U(t) \\
& \quad+\lambda_{1} \sum_{i=1}^{k} b_{i}(t) U\left(\tau_{i}(t)\right)-\sum_{i=1}^{m} p_{i}(t) \varphi_{i}\left(U\left(\sigma_{i}(t)\right)\right) \geq G(t), \quad t \geq t_{2} .
\end{aligned}
$$

It is easy to check that

$$
|U(t)| \leq K_{\Phi} \int_{G}|u(x, t)| \Phi(x) d x \leq K_{\Phi} \int_{G} \gamma(x, t) \Phi(x) d x=\Gamma(t),
$$

and therefore $U(t) \in \tilde{\mathcal{B}}_{\Gamma}$. Hence, $\left(2_{+}\right)$has an eventually positive solution $U(t)$ of class $\tilde{\mathcal{B}}_{\Gamma}$. This contradicts the hypothesis. If $u<0$ in $G \times\left[t_{0}, \infty\right)$, it can be shown that

$$
c\left(x, t,\left(z_{i}[u](x, t)\right)_{i=1}^{M}\right) \leq-\sum_{i=1}^{m} p_{i}(t) \varphi_{i}\left(-u\left(x, \sigma_{i}(t)\right)\right) \quad \text { in } \quad G \times\left[t_{1}, \infty\right)
$$

for some $t_{1} \geq t_{0}$. Letting $v \equiv-u$, we obtain

$$
\begin{aligned}
& \frac{\partial}{\partial t}\left(v(x, t)+\sum_{i=1}^{\ell} h_{i}(t) v\left(x, \rho_{i}(t)\right)\right) \\
& \quad-a(t) \Delta v(x, t)-\sum_{i=1}^{k} b_{i}(t) \Delta v\left(x, \tau_{i}(t)\right) \\
& \quad-\sum_{i=1}^{m} p_{i}(t) \varphi_{i}\left(v\left(x, \sigma_{i}(t)\right)\right) \geq-f(x, t) \quad \text { in } \quad G \times\left[t_{1}, \infty\right) .
\end{aligned}
$$

Proceeding as in the case where $u>0$, we are led to a contradiction. The proof is complete.

Theorem 2. If the functional differential inequalities $\left(8_{ \pm}\right)$

$$
\frac{d}{d t}\left(y(t)+\sum_{i=1}^{\ell} h_{i}(t) y\left(\rho_{i}(t)\right)\right)-\sum_{i=1}^{m} p_{i}(t) \varphi_{i}\left(y\left(\sigma_{i}(t)\right)\right) \geq \pm \tilde{G}(t)
$$


have no eventually positive (bounded) solutions, then every (bounded) solution $u$ of the boundary value problem $(1),\left(B_{2}\right)$ is oscillatory in $\Omega$, where

$$
\tilde{G}(t)=\tilde{F}(t)+a(t) \tilde{\Psi}(t)+\sum_{i=1}^{k} b_{i}(t) \tilde{\Psi}\left(\tau_{i}(t)\right) .
$$

Proof. Assume on the contrary, that there exists a (bounded) solution $u$ of the problem (1), $\left(\mathrm{B}_{2}\right)$ such that $u>0$ in $G \times\left[t_{0}, \infty\right)$ for some $t_{0}>0$. Arguing as in the proof of Theorem 1, we observe that the inequality (3) holds for some $t_{1} \geq t_{0}$. Dividing (3) by $|G|$ and then integrating over $G$ yields

$$
\begin{aligned}
& \frac{d}{d t}\left(\tilde{U}(t)+\sum_{i=1}^{\ell} h_{i}(t) \tilde{U}\left(\rho_{i}(t)\right)\right)-a(t) \frac{1}{|G|} \int_{G} \Delta u(x, t) d x \\
& \quad-\sum_{i=1}^{k} b_{i}(t) \frac{1}{|G|} \int_{G} \Delta u\left(x, \tau_{i}(t)\right) d x-\sum_{i=1}^{m} p_{i}(t) \frac{1}{|G|} \int_{G} \varphi_{i}\left(u\left(x, \sigma_{i}(t)\right)\right) d x \\
& \geq \tilde{F}(t), \quad t \geq t_{1} .
\end{aligned}
$$

It follows from the divergence theorem that

$$
\begin{aligned}
\frac{1}{|G|} \int_{G} \Delta u(x, t) d x & =\frac{1}{|G|} \int_{\partial G} \frac{\partial u}{\partial \nu}(x, t) d S \\
& =\frac{1}{|G|} \int_{\partial G}(\mu u(x, t)+\tilde{\psi}) d S \\
& \geq \tilde{\Psi}(t), \quad t \geq t_{1} .
\end{aligned}
$$

Analogously we obtain

$$
\frac{1}{|G|} \int_{G} \Delta u\left(x, \tau_{i}(t)\right) d x \geq \tilde{\Psi}\left(\tau_{i}(t)\right), \quad t \geq t_{2}
$$

for some $t_{2} \geq t_{1}$. An application of Jensen's inequality shows that

$$
\frac{1}{|G|} \int_{G} \varphi_{i}\left(u\left(x, \sigma_{i}(t)\right)\right) d x \geq \varphi_{i}\left(\tilde{U}\left(\sigma_{i}(t)\right)\right), \quad t \geq t_{2} .
$$

Combining (9)-(12) yields

$$
\frac{d}{d t}\left(\tilde{U}(t)+\sum_{i=1}^{\ell} h_{i}(t) \tilde{U}\left(\rho_{i}(t)\right)\right)-\sum_{i=1}^{m} p_{i}(t) \varphi_{i}\left(\tilde{U}\left(\sigma_{i}(t)\right)\right) \geq \tilde{G}(t), \quad t \geq t_{2},
$$

which means that $\tilde{U}(t)$ is an eventually positive (bounded) solution of $\left(8_{+}\right)$. This contradicts the hypothesis. The case where $u<0$ can be treated similarly, and we are led to a contradiction. The proof is complete. 


\section{Functional differential inequalities}

In this section we investigate the nonexistence of eventually positive solutions of the functional differential inequality

$$
\frac{d}{d t}\left(y(t)+\sum_{i=1}^{\ell} h_{i}(t) y\left(\rho_{i}(t)\right)\right)-\sum_{i=1}^{m} p_{i}(t) \varphi_{i}\left(y\left(\sigma_{i}(t)\right)\right) \geq G(t)
$$

Theorem 3. Assume that $\sum_{i=1}^{\ell} h_{i}(t) \leq 1, \rho_{i}(t) \leq t(i=1,2, \ldots, \ell)$, and $\varphi_{j}(s)$ is nondecreasing on $[0, \infty)$ for some $j \in\{1,2, \ldots, m\}$. The inequality (13) has no eventually positive bounded solution if there is a function $\Theta(t) \in$ $C^{1}((0, \infty) ; \mathbf{R})$ such that $\Theta(t)$ is bounded and oscillatory at $t=\infty, \Theta^{\prime}(t)=$ $G(t)$, and

$$
\begin{aligned}
\int_{s_{0}}^{\infty} p_{j}(s) \varphi_{j}( & {\left[\left(1-\sum_{i=1}^{\ell} h_{i}\left(\sigma_{j}(s)\right)\right)\left[\Theta\left(\sigma_{j}(s)\right)\right]_{-}\right.} \\
& \left.\left.+\Theta\left(\sigma_{j}(s)\right)-\sum_{i=1}^{\ell} h_{i}\left(\sigma_{j}(s)\right) \Theta\left(\rho_{i}\left(\sigma_{j}(s)\right)\right)\right]_{+}\right) d s=\infty
\end{aligned}
$$

for some $s_{0}>0$, where

$$
[\Theta(t)]_{ \pm}=\max \{ \pm \Theta(t), 0\} .
$$

Proof. Assume on the contrary, that there exists an eventually positive bounded solution $y(t)$ of $(13)$ such that $y(t)>0$ on $\left[t_{0}, \infty\right)$ for some $t_{0}>0$. Then, $y\left(\rho_{i}(t)\right)>0(i=1,2, \ldots, \ell), y\left(\sigma_{i}(t)\right)>0(i=1,2, \ldots, m)$ on $\left[t_{1}, \infty\right)$ for some $t_{1} \geq t_{0}$. Letting

$$
z(t)=y(t)+\sum_{i=1}^{\ell} h_{i}(t) y\left(\rho_{i}(t)\right)-\Theta(t)
$$

we see that

$$
z^{\prime}(t) \geq \sum_{i=1}^{m} p_{i}(t) \varphi_{i}\left(y\left(\sigma_{i}(t)\right)\right) \geq 0, \quad t \geq t_{1}
$$

and therefore $z(t)$ is nondecreasing for $t \geq t_{1}$. Hence, we find that either $z(t)>0$ or $z(t) \leq 0$ on $\left[t_{2}, \infty\right)$ for some $t_{2} \geq t_{1}$. If $z(t) \leq 0$ on $\left[t_{2}, \infty\right)$, then

$$
y(t)+\sum_{i=1}^{\ell} h_{i}(t) y\left(\rho_{i}(t)\right) \leq \Theta(t), \quad t \geq t_{2} .
$$

The left hand side of (15) is positive, but the right hand side of (15) is oscillatory at $t=\infty$. This is a contradiction. Hence, we conclude that 
$z(t)>0$ on $\left[t_{2}, \infty\right)$. Since $z(t)+\Theta(t)>0$ on $\left[t_{2}, \infty\right)$, we find that $z(t)>$ $-\Theta(t)$ on $\left[t_{2}, \infty\right)$, and therefore

$$
z(t) \geq[\Theta(t)]_{-} \quad \text { for } \quad t \geq t_{2}
$$

In view of the fact that $y(t) \leq z(t)+\Theta(t)$ and $z(t)$ is nondecreasing, we obtain

$$
\begin{aligned}
(17) y(t) & =z(t)-\sum_{i=1}^{\ell} h_{i}(t) y\left(\rho_{i}(t)\right)+\Theta(t) \\
& \geq z(t)-\sum_{i=1}^{\ell} h_{i}(t)\left(z\left(\rho_{i}(t)\right)+\Theta\left(\rho_{i}(t)\right)\right)+\Theta(t) \\
& \geq\left(1-\sum_{i=1}^{\ell} h_{i}(t)\right) z(t)+\Theta(t)-\sum_{i=1}^{\ell} h_{i}(t) \Theta\left(\rho_{i}(t)\right), \quad t \geq t_{2} .
\end{aligned}
$$

Combining (16) with (17) yields

$$
y(t) \geq\left(1-\sum_{i=1}^{\ell} h_{i}(t)\right)[\Theta(t)]_{-}+\Theta(t)-\sum_{i=1}^{\ell} h_{i}(t) \Theta\left(\rho_{i}(t)\right), \quad t \geq t_{2} .
$$

Since $y(t)>0$ for $t \geq t_{2}$, we observe that

$$
\begin{aligned}
& y\left(\sigma_{j}(t)\right) \geq \\
& {\left[\left(1-\sum_{i=1}^{\ell} h_{i}\left(\sigma_{j}(t)\right)\right)\left[\Theta\left(\sigma_{j}(t)\right)\right]_{-}+\Theta\left(\sigma_{j}(t)\right)-\sum_{i=1}^{\ell} h_{i}\left(\sigma_{j}(t)\right) \Theta\left(\rho_{i}\left(\sigma_{j}(t)\right)\right)\right]_{+}}
\end{aligned}
$$

on $\left[t_{3}, \infty\right)$ for some $t_{3} \geq t_{2}$. Hence, we obtain

$$
\begin{aligned}
(18) z^{\prime}(t) & \geq \sum_{i=1}^{m} p_{i}(t) \varphi_{i}\left(y\left(\sigma_{i}(t)\right)\right) \\
& \geq p_{j}(t) \varphi_{j}\left(y\left(\sigma_{j}(t)\right)\right) \\
& \geq p_{j}(t) \varphi_{j}\left(\left[\left(1-\sum_{i=1}^{\ell} h_{i}\left(\sigma_{j}(t)\right)\right)\left[\Theta\left(\sigma_{j}(t)\right)\right]_{-}\right.\right. \\
& \left.\left.\quad+\Theta\left(\sigma_{j}(t)\right)-\sum_{i=1}^{\ell} h_{i}\left(\sigma_{j}(t)\right) \Theta\left(\rho_{i}\left(\sigma_{j}(t)\right)\right)\right]_{+}\right), \quad t \geq t_{3} .
\end{aligned}
$$


Integrating (18) over $\left[t_{3}, t\right]$ yields

$$
\begin{aligned}
(19) z(t)-z\left(t_{3}\right) & \\
\geq \int_{t_{3}}^{t} p_{j}(s) \varphi_{j}( & {\left[\left(1-\sum_{i=1}^{\ell} h_{i}\left(\sigma_{j}(s)\right)\right)\left[\Theta\left(\sigma_{j}(s)\right)\right]_{-}\right.} \\
+ & \left.\left.\Theta\left(\sigma_{j}(s)\right)-\sum_{i=1}^{\ell} h_{i}\left(\sigma_{j}(s)\right) \Theta\left(\rho_{i}\left(\sigma_{j}(s)\right)\right)\right]_{+}\right) d s, \quad t \geq t_{3} .
\end{aligned}
$$

The left hand side of (19) is bounded from above, but the right hand side of (19) tends to infinity as $t \rightarrow \infty$. This is a contradiction and the proof is complete.

Next we consider the functional differential inequality

$$
y^{\prime}(t)-p(t) y(\sigma(t)) \geq q(t), \quad t \geq T,
$$

where $T$ is some positive number, $p(t) \in C([T, \infty) ;[0, \infty)), q(t) \in C([T, \infty)$; $\mathbf{R})$ and $\sigma(t) \in C([T, \infty) ; \mathbf{R})$ for which $\lim _{t \rightarrow \infty} \sigma(t)=\infty, \sigma(t) \geq t$ and $\sigma(t)$ is nondecreasing on $[T, \infty)$.

Lemma 1. The inequality (20) has no eventually positive solution if there exists a sequence $\left\{t_{n}\right\}$ such that:

$$
\begin{aligned}
& \lim _{n \rightarrow \infty} t_{n}=\infty \\
& \int_{t_{n}}^{\sigma\left(t_{n}\right)} p(s) d s \geq 1, \\
& \int_{t_{n}}^{\sigma\left(t_{n}\right)} q(s) d s+\int_{t_{n}}^{\sigma\left(t_{n}\right)} p(s)\left(\int_{\sigma\left(t_{n}\right)}^{\sigma(s)} q(\xi) d \xi\right) d s \geq 0 .
\end{aligned}
$$

Proof. Suppose that there exists a solution $y(t)$ of $(20)$ for which $y(t)>0$ on $\left[T_{0}, \infty\right)$ for some $T_{0}>T$. Integrating $(20)$ over $[t, \sigma(t)]$, we obtain

$$
y(\sigma(t))-y(t)-\int_{t}^{\sigma(t)} p(s) y(\sigma(s)) d s \geq \int_{t}^{\sigma(t)} q(s) d s, \quad t \geq T_{0} .
$$

Since

$$
y^{\prime}(t) \geq q(t) \quad \text { for } \quad t \geq T_{0}
$$

an integration of $(22)$ over $[\sigma(t), \sigma(s)]$ yields

$$
y(\sigma(s))-y(\sigma(t)) \geq \int_{\sigma(t)}^{\sigma(s)} q(\xi) d \xi \quad \text { for } \quad s \geq t,
$$


and therefore

$$
y(\sigma(s)) \geq y(\sigma(t))+\int_{\sigma(t)}^{\sigma(s)} q(\xi) d \xi \quad \text { for } \quad s \geq t .
$$

Combining (21) with (23), we obtain

$y(\sigma(t))-y(t)-\int_{t}^{\sigma(t)} p(s)\left(y(\sigma(t))+\int_{\sigma(t)}^{\sigma(s)} q(\xi) d \xi\right) d s \geq \int_{t}^{\sigma(t)} q(s) d s, t \geq T_{0}$,

or equivalently

$$
\begin{aligned}
& -y(t)-y(\sigma(t))\left(\int_{t}^{\sigma(t)} p(s) d s-1\right) \\
\geq & \int_{t}^{\sigma(t)} q(s) d s+\int_{t}^{\sigma(t)} p(s)\left(\int_{\sigma(t)}^{\sigma(s)} q(\xi) d \xi\right) d s, \quad t \geq T_{0} .
\end{aligned}
$$

It is easy to see that $t_{n} \geq T_{0}(n \geq N)$ for some positive integer $N$. We easily see that the left hand side of $(24)$ with $t=t_{n}(n \geq N)$ is negative, whereas the right hand side of $(24)$ with $t=t_{n}(n \geq N)$ is nonnegative. This is a contradiction and the proof is complete.

Theorem 4. Assume that $\sum_{i=1}^{\ell} h_{i}(t) \leq 1, \rho_{i}(t) \leq t(i=1,2, \ldots, \ell)$, and $\varphi_{j}(s) \geq \beta s$ in $(0, \infty)$ for some $\beta>0$ and some $j \in\{1,2, \ldots, m\}$. Moreover, assume that $\sigma_{j}(t) \geq t$ and $\sigma_{j}(t)$ is nondecreasing in $(0, \infty)$, and that there is a function $\Theta(t) \in C^{1}((0, \infty) ; \mathbf{R})$ such that $\Theta(t)$ is oscillatory at $t=\infty$ and $\Theta^{\prime}(t)=G(t)$. The inequality (13) has no eventually positive solution if there exists a sequence $\left\{t_{n}\right\}$ for which

$$
\begin{gathered}
\lim _{n \rightarrow \infty} t_{n}=\infty \\
\int_{t_{n}}^{\sigma_{j}\left(t_{n}\right)} p_{j}(s)\left(1-\sum_{i=1}^{\ell} h_{i}\left(\sigma_{j}(s)\right)\right) d s \geq \frac{1}{\beta}, \\
\int_{t_{n}}^{\sigma_{j}\left(t_{n}\right)} Q(s) d s+ \\
\beta \int_{t_{n}}^{\sigma_{j}\left(t_{n}\right)} p_{j}(s)\left(1-\sum_{i=1}^{\ell} h_{i}\left(\sigma_{j}(s)\right)\right)\left(\int_{\sigma_{j}\left(t_{n}\right)}^{\sigma_{j}(s)} Q(\xi) d \xi\right) d s \geq 0,
\end{gathered}
$$

where

$$
Q(t)=\beta p_{j}(t)\left(\Theta\left(\sigma_{j}(t)\right)-\sum_{i=1}^{\ell} h_{i}\left(\sigma_{j}(t)\right) \Theta\left(\rho_{i}\left(\sigma_{j}(t)\right)\right)\right)
$$


Proof. Let $y(t)$ be a solution of $(13)$ such that $y(t)>0$ on $\left[t_{0}, \infty\right)$ for some $t_{0}>0$. Proceeding as in the proof of Theorem 3, we see that (17) holds, and hence we obtain

$$
\begin{aligned}
y\left(\sigma_{j}(t)\right) \geq\left(1-\sum_{i=1}^{\ell} h_{i}\left(\sigma_{j}(t)\right)\right) z\left(\sigma_{j}(t)\right) \\
\quad+\Theta\left(\sigma_{j}(t)\right)-\sum_{i=1}^{\ell} h_{i}\left(\sigma_{j}(t)\right) \Theta\left(\rho_{i}\left(\sigma_{j}(t)\right)\right), \quad t \geq t_{3}
\end{aligned}
$$

for some $t_{3} \geq t_{2}$. Then it can be shown that

$$
\begin{aligned}
z^{\prime}(t) & \geq \sum_{i=1}^{m} p_{i}(t) \varphi_{i}\left(y\left(\sigma_{i}(t)\right)\right) \\
& \geq p_{j}(t) \varphi_{j}\left(y\left(\sigma_{j}(t)\right)\right) \\
& \geq \beta p_{j}(t) y\left(\sigma_{j}(t)\right), \quad t \geq t_{3} .
\end{aligned}
$$

Combining (28) with (29), we observe that $z(t)$ is a positive solution of

$$
z^{\prime}(t)-\beta p_{j}(t)\left(1-\sum_{i=1}^{\ell} h_{i}\left(\sigma_{j}(t)\right)\right) z\left(\sigma_{j}(t)\right) \geq Q(t)
$$

for $t \geq t_{3}$. However, Lemma 1 implies that (30) has no eventually positive solution. This is a contradiction and the proof is complete.

\section{Functional parabolic equations}

Combining the results in Sections 2 and 3, we can derive various oscillation theorems for the boundary value problems $(1),\left(\mathrm{B}_{i}\right)(i=1,2)$.

Lemma 2. If $\left(2_{ \pm}\right)$have eventually positive solutions $y_{r}(t) \in \tilde{\mathcal{B}}_{\Gamma}(r=1,2)$, respectively, then $y_{r}(t)$ are eventually positive solutions of the differential inequalities

$$
\frac{d}{d t}\left(y(t)+\sum_{i=1}^{\ell} h_{i}(t) y\left(\rho_{i}(t)\right)\right)-\sum_{i=1}^{m} p_{i}(t) \varphi_{i}\left(y\left(\sigma_{i}(t)\right)\right) \geq G_{r}(t),
$$

where

$$
G_{r}(t)=(-1)^{r-1} G(t)-\lambda_{1} a(t) \Gamma(t)-\lambda_{1} \sum_{i=1}^{k} b_{i}(t) \Gamma\left(\tau_{i}(t)\right) \quad(r=1,2) .
$$


Proof. Since

$$
\lambda_{1} a(t) y_{r}(t) \leq \lambda_{1} a(t) \Gamma(t), \quad \lambda_{1} \sum_{i=1}^{k} b_{i}(t) y_{r}\left(\tau_{i}(t)\right) \leq \lambda_{1} \sum_{i=1}^{k} b_{i}(t) \Gamma\left(\tau_{i}(t)\right),
$$

we easily see that $y_{r}(t)$ are eventually positive solutions of $(31)$.

Theorem 5. Assume that $\sum_{i=1}^{\ell} h_{i}(t) \leq 1, \rho_{i}(t) \leq t(i=1,2, \ldots, \ell), \varphi_{j}(s)$ is nondecreasing on $[0, \infty)$ for some $j \in\{1,2, \ldots, m\}$. Every solution $u \in \mathcal{B}_{K}$ ( $K$ is a positive constant ) of the boundary value problem $(1),\left(B_{1}\right)$ is oscillatory in $\Omega$ if there is a function $\Theta_{r}(t) \in C^{1}((0, \infty) ; \mathbf{R})(r=1,2)$ such that $\Theta_{r}(t)$ is bounded and oscillatory at $t=\infty, \Theta_{r}^{\prime}(t)=G_{r}(t)$ with $\Gamma(t) \equiv K$, and that

$$
\begin{aligned}
\int_{s_{0}}^{\infty} p_{j}(s) \varphi_{j}( & {\left[\left(1-\sum_{i=1}^{\ell} h_{i}\left(\sigma_{j}(s)\right)\right)\left[\Theta_{r}\left(\sigma_{j}(s)\right)\right]_{-}\right.} \\
& \left.\left.+\Theta_{r}\left(\sigma_{j}(s)\right)-\sum_{i=1}^{\ell} h_{i}\left(\sigma_{j}(s)\right) \Theta_{r}\left(\rho_{i}\left(\sigma_{j}(s)\right)\right)\right]_{+}\right) d s=\infty
\end{aligned}
$$

for some $s_{0}>0$.

Proof. It follows from Theorem 3 that (31) have no eventually positive bounded solutions, and hence Lemma 2 with $\gamma(x, t)=\Gamma(t)=K$ implies that $\left(2_{ \pm}\right)$have no eventually positive solutions $y(t) \in \tilde{\mathcal{B}}_{K}$. The conclusion follows from Theorem 1.

Theorem 6. Assume that $\sum_{i=1}^{\ell} h_{i}(t) \leq 1, \quad \rho_{i}(t) \leq t(i=1,2, \ldots, \ell)$, and $\varphi_{j}(s) \geq \beta s$ in $(0, \infty)$ for some $\beta>0$ and some $j \in\{1,2, \ldots, m\}$. Moreover, assume that $\sigma_{j}(t) \geq t$ and $\sigma_{j}(t)$ is nondecreasing in $(0, \infty)$, and that there is a function $\Theta_{r}(t) \in C^{1}((0, \infty) ; \mathbf{R})(r=1,2)$ such that $\Theta_{r}(t)$ is oscillatory at $t=\infty$ and $\Theta_{r}^{\prime}(t)=G_{r}(t)$. Every solution $u \in \mathcal{B}_{\gamma}(\Omega)$ of the boundary value problem (1), (B $\left.B_{1}\right)$ is oscillatory in $\Omega$ if there exists a sequence $\left\{t_{r, n}\right\} \quad(r=$ $1,2)$ for which $(25)-(27)$ with $t_{n}=t_{r, n}$ and $Q(t)$ replaced by

$$
Q_{r}(t)=\beta p_{j}(t)\left(\Theta_{r}\left(\sigma_{j}(t)\right)-\sum_{i=1}^{\ell} h_{i}\left(\sigma_{j}(t)\right) \Theta_{r}\left(\rho_{i}\left(\sigma_{j}(t)\right)\right)\right)
$$

hold.

Proof. Theorem 4 implies that (31) have no eventually positive solutions. Hence, it follows from Lemma 2 that $\left(2_{ \pm}\right)$have no eventually positive solutions $y(t) \in \tilde{\mathcal{B}}_{\Gamma}$. The conclusion follows from Theorem 1 . 
Theorem 7. Assume that $\sum_{i=1}^{\ell} h_{i}(t) \leq 1, \rho_{i}(t) \leq t(i=1,2, \ldots, \ell), \varphi_{j}(s)$ is nondecreasing on $[0, \infty)$ for some $j \in\{1,2, \ldots, m\}$. Every bounded solution $u$ of the boundary value problem $(1),\left(B_{2}\right)$ is oscillatory in $\Omega$ if there is a function $\Theta(t) \in C^{1}((0, \infty) ; \mathbf{R})$ such that $\Theta(t)$ is bounded and oscillatory at $t=\infty, \Theta^{\prime}(t)=\tilde{G}(t)$, and that

$$
\begin{aligned}
\int_{s_{0}}^{\infty} p_{j}(s) \varphi_{j}( & {\left[\left(1-\sum_{i=1}^{\ell} h_{i}\left(\sigma_{j}(s)\right)\right)\left[\Theta\left(\sigma_{j}(s)\right)\right]_{\mp}\right.} \\
& \left.\left. \pm\left(\Theta\left(\sigma_{j}(s)\right)-\sum_{i=1}^{\ell} h_{i}\left(\sigma_{j}(s)\right) \Theta\left(\rho_{i}\left(\sigma_{j}(s)\right)\right)\right)\right]_{+}\right) d s=\infty
\end{aligned}
$$

for some $s_{0}>0$.

Proof. Combining Theorem 2 with Theorem 3, we are led to the conclusion.

Theorem 8. Assume that $\sum_{i=1}^{\ell} h_{i}(t) \leq 1, \quad \rho_{i}(t) \leq t(i=1,2, \ldots, \ell)$, and $\varphi_{j}(s) \geq \beta s$ in $(0, \infty)$ for some $\beta>0$ and some $j \in\{1,2, \ldots, m\}$. Moreover, assume that $\sigma_{j}(t) \geq t$ and $\sigma_{j}(t)$ is nondecreasing in $(0, \infty)$, and that there is a function $\Theta(t) \in C^{1}((0, \infty) ; \mathbf{R})$ such that $\Theta(t)$ is oscillatory at $t=\infty$ and $\Theta^{\prime}(t)=\tilde{G}(t)$. Every solution $u$ of the boundary value problem (1), (B $\left.B_{2}\right)$ is oscillatory in $\Omega$ if there exists a sequence $\left\{t_{n}\right\}$ satisfying (25), (26) and

$$
\int_{t_{n}}^{\sigma_{j}\left(t_{n}\right)} Q(s) d s+\beta \int_{t_{n}}^{\sigma_{j}\left(t_{n}\right)} p_{j}(s)\left(1-\sum_{i=1}^{\ell} h_{i}\left(\sigma_{j}(s)\right)\right)\left(\int_{\sigma_{j}\left(t_{n}\right)}^{\sigma_{j}(s)} Q(\xi) d \xi\right) d s=0 .
$$

Proof. The conclusion follows by combining Theorem 2 with Theorem 4 .

We conclude with an example which illustrates Theorem 7 .

Example. We consider the problem

$$
\begin{aligned}
& \frac{\partial}{\partial t}(u(x, t)+(1 / 2) u(x, t+\pi)) \\
& -u_{x x}(x, t)-u_{x x}(x, t+\pi)-u(x, t+(\pi / 2)) \\
& =-(\cos x+1) \cos t, \quad(x, t) \in(0, \pi) \times(0, \infty), \\
& \quad-u_{x}(0, t)=u_{x}(\pi, t)=0, \quad t>0 .
\end{aligned}
$$

Here $n=1, G=(0, \pi), \Omega=(0, \pi) \times(0, \infty), \ell=k=m=M=1$, $h_{1}(t)=1 / 2, \rho_{1}(t)=t+\pi, a(t)=1, b_{1}(t)=1, \tau_{1}(t)=t+\pi, p_{1}(t)=1$, 
$\sigma_{1}(t)=t+(\pi / 2), \varphi_{1}(s)=s, \mu \equiv 0, \tilde{\psi} \equiv 0$ and $f(x, t)=-(\cos x+1) \cos t$. It is easily seen that $\tilde{\Psi}(t) \equiv 0$ and

$$
\tilde{G}(t)=\tilde{F}(t)=\frac{1}{\pi} \int_{0}^{\pi} f(x, t) d x=-\cos t .
$$

Choosing $\Theta(t)=-\sin t$, we see that $\Theta(t) \in C^{1}((0, \infty) ; \mathbf{R}), \Theta^{\prime}(t)=\tilde{G}(t)$, $\Theta(t)$ is bounded and oscillatory at $t=\infty$. It is easy to check that $\Theta\left(\sigma_{1}(s)\right)=$ $-\cos s, \Theta\left(\rho_{1}\left(\sigma_{1}(s)\right)\right)=\cos s$, and that

$$
\begin{aligned}
& \int_{s_{0}}^{\infty}\left[\left(1-\frac{1}{2}\right)[-\cos s]_{\mp} \pm\left(-\cos s-\frac{1}{2} \cos s\right)\right]_{+} d s \\
= & \frac{1}{2} \int_{s_{0}}^{\infty}\left[[-\cos s]_{\mp} \pm(-3 \cos s)\right]_{+} d s \\
= & \frac{1}{2} \int_{s_{0}}^{\infty}[\mp 3 \cos s]_{+} d s=\infty .
\end{aligned}
$$

Hence, it follows from Theorem 7 that every bounded solution $u$ of the problem $(32),(33)$ is oscillatory in $(0, \pi) \times(0, \infty)$. One such solution is $u=2(\cos x+1) \sin t$.

Acknowledgment. The authors would like to thank the referee for his helpful comments and suggestions.

\section{References}

[1] Bainov, D.D. and Minchev, E.I., Forced oscillations of solutions of impulsive nonlinear parabolic equations, J. Appl. Anal. (to appear).

[2] Bykov, Ya.V. and Kultaev, T.Ch., Oscillation of solutions of a class of parabolic equations, Izv. Akad. Nauk Kirgiz. SSR 6 (1983), 3-9 (Russian).

[3] Courant, R. and Hilbert, D., Methods of Mathematical Physics, Vol. I, Interscience, New York, 1966.

[4] Cui, B.T., Oscillation theorems of nonlinear parabolic equations of neutral type, Math. J. Toyama Univ. 14 (1991), 113-123.

[5] Mishev, D.P., Oscillation of the solutions of hyperbolic differential equations of neutral type with "maxima", Godishnik Vissh. Uchebn. Zaved. Prilozhna Mat. 25 (1989), 918 .

[6] Mishev, D.P. and Bainov, D.D., Oscillation of the solutions of parabolic differential equations of neutral type, Appl. Math. Comput. 28 (1988), 97-111.

[7] Okikiolu, G.O., Aspects of the Theory of Bounded Integral Operators in $L^{p}$-spaces, Academic Press, New York, 1971.

[8] Tanaka, S. and Yoshida, N., Oscillations of solutions to parabolic equations with deviating arguments, Tamkang J. Math. 28 (1997), 169-181.

[9] Yoshida, N., On the oscillation of solutions to parabolic equations with functional arguments, Math. J. Toyama Univ. 18 (1995), 65-78. 
[10] Yoshida, N., Forced oscillations of nonlinear parabolic equations with functional arguments, Analysis 15 (1995), 71-84.

TAKAŜI KUSANO

Department of Applied Mathematics

Faculty of Science

FUKUOKA UNIVERSITY

FUKUOKA 814-0180

JAPAN
Norio YoshidA

Department of Mathematics FACUlTy OF SCIENCE TOYAMA UNIVERSITY TOYAMA 930-8555

JAPAN 